\title{
Impaired right ventricular filling in patients with a chronic coronary syndrome
}

\author{
Alexey N. Sumin, Ekaterina V. Korok, Tatjana Ju. Sergeeva
}

Federal State Budgetary Institution "Research Institute for Complex Issues of Cardiovascular Disease", Kemerovo, Russian Federation

\begin{abstract}
Aim: The prognostic value of right ventricle (RV) systolic dysfunction is known but the RV diastolic dysfunction (RVDD) is less investigated, thus the purpose of this study was to assess the incidence of RVDD in patients with coronary artery disease (CAD) and to identify factors associated with its presence. Material and methods: We examined 200 patients with stable CAD (153 men). RV diastolic parameters were studied by echocardiography: peak velocity of early (Et) and late (At) RV filling and their ratio, velocities at the tricuspid annulus (et', a't, s't). Patients were divided into 2 groups: with (n=92) and without RVDD ( $n=108)$. Results: Old myocardial infarction (MI) $(p=0.007)$, anterior MI $(p=0.001)$, congestive heart failure $(p=0.030)$ and peripheral arterial disease $(\mathrm{p}=0.030)$ were more prevalent in patients with RVDD. The end-systolic dimensions were higher $(\mathrm{p}=0.010)$, while left ventricular $(\mathrm{LV})$ ejection fraction $(\mathrm{EF})(\mathrm{p}=0.044)$ and the mitral $\mathrm{E} / \mathrm{A}$ ratio $(\mathrm{p}<0.001)$ were lower in this group. No significant differences were found between the groups in the coronary artery stenosis. Independent predictors of the RVDD presence were increased age, old MI, hyperlipidemia, moderate chronic heart failure, peripheral arterial disease, and decreased LVEF. Conclusions: The RV diastolic dysfunction is much more common than systolic dysfunction in patients with stable CAD (46\% and 7.5\%, respectively). The RVDD presence was predominantly associated with an increase in age and LV systolic dysfunction, but not with coronary artery lesions. The predictive value of RVDD requires further research.
\end{abstract}

Keywords: right ventricular; diastolic dysfunction; coronary artery disease

\section{Introduction}

The right heart's dysfunction has been the point of extensive research over recent years. These studies have mostly investigated the patients with congenital heart disease, acquired valvular heart disease, pulmonary hypertension and advanced heart failure [1-4]. Influence of myocardial infarction on the right ventricle function [5]

Received 23.07.2020 Accepted 16.12.2020

Med Ultrason

2021, Vol. 23, No 3, 311-318

Corresponding author: Sumin Alexey N. - MD, PhD

Laboratory of Comorbidity in Cardiovascular

Diseases, Federal State Budgetary

Institution "Research Institute for Complex

Issues of Cardiovascular Disease"

6 Sosnoviy Blvd, 650002, Kemerovo,

Russian Federation

Phone: (3842) 64-44-61, 8-903-940-8668

Fax: (3842) 64-27-18

E-mail: an_sumin@mail.ru and perioperative right ventricle condition [6] have been the main focus of research among patients with coronary artery disease (CAD). One of the major reasons leading to this increase of attention is the improved diagnostic capacity due to ultrasound cardiac imaging advancements, including three-dimensional (3D) right ventricle evaluation [7]. The other is proven clinical and prognostic value of right ventricle evaluation in different clinical scenarios [7-9].

Nearly all data on the importance of RV function is notably focused on its systolic dysfunction while RV filling is far less studied, perhaps because of challenges in diastolic dysfunction assessment. Nevertheless, several experiments have shown that the development of RV diastolic dysfunction can precede systolic dysfunction in pulmonary hypertension [10] and a diabetes model [11]. Independent prognostic value of RV diastolic dysfunction has been identified in certain clinical situations $[1,12]$ but these studies are few in number. Therefore, it is important 
to continue investigating the RV diastolic function and clarify its possible clinical and prognostic significance in various groups of patients. All these facts led us to perform this study that aimed at assessing the incidence of $\mathrm{RV}$ diastolic dysfunction in patients with stable CAD and identifying factors associated with its presence.

\section{Material and methods}

\section{Study population}

This one-center cross-sectional study involved CAD patients admitted to the cardiology department of Research Institute for Complex Issues of Cardiovascular Disease before coronary artery bypass grafting $(\mathrm{CABG})$ from February 2017 to November 2018. The study excluded patients with recent acute coronary syndrome, respiratory failure due to pulmonary disease, exacerbation of underlying or concomitant disease, valvular heart disease, malignant tumors, left ventricle ejection fraction $\leq 40 \%$ or poor visualization during ultrasound examination. Patients' enrolment in the study was discontinued after reaching the amount of 200 patients. The study was carried out in accordance with the Helsinki Declaration, approved by the Local Ethics Committee and all study participants signed an informed consent.

\section{Data collection and study outcomes}

This study focused on echocardiographic parameters in the stable CAD patients who underwent a transthoracic echocardiogram before CABG. Detailed patient demographics and clinical characteristics were collected from the electronic database of the institute's CABG registry, including a history of cardiovascular disease (angina pectoris, myocardial infarction history, arrhythmias, prior percutaneous coronary intervention, heart failure symptoms, peripheral vascular disease, stroke), cardiac risk factors (hypertension, hyperlipidaemia, diabetes, smoking), chronic pulmonary disease and medical therapies, were recorded for each patient. The invasive coronary angiography (ICA) was performed within 6 months before index admission. The ICA results are obtained from the clinic's electronic database.

The primary outcome measure was the comparative frequency of RV systolic and diastolic dysfunction (RVDD) in CAD patients. Then we compared the groups with and without RVDD by demographic, clinical, echocardiographic and angiographic variables. We also assessed the factors associated with the RVDD presence using logistic regression analysis.

\section{Echocardiographic examination}

Standard transthoracic echocardiography was performed on a Philips Clear Vue 550 ultrasound scanner (USA). The images were obtained using the long and short axes in the parasternal and apical projections and all measurements were performed according to current recommendations [13]. All echocardiographic examinations were performed by the same examiner (TS) with 30 years of experience. Reported values of all echocardiographic parameters were obtained as the average value of 3 consecutive cardiac cycles. A random sample of 50 patients was reviewed by another examiner (32 years of experience) who was blind to the initial measurement performed by the sonographer. Diameter and thickness of the LV walls were measured in the twodimensional M-mode in accordance with basic principles of international recommendations. Left heart analysis included assessment of the size and volumetric parameters of the left ventricle, stroke volume, left ventricular mass, the maximum transverse diameter of the left atrium in the diastole (LA) and its volume (LAV). Ejection fraction of the left ventricle (LVEF) was calculated according to the Simpson method. Analyzing the right heart was performed by assessing the right atrium (RA) size, right ventricle (RV) size and RV wall thickness. Tricuspid annular plane systolic excursion (TAPSE) was measured at the lateral tricuspid annulus with M-mode.

In pulsed-wave Doppler imaging, LV diastolic parameters were studied: peak velocity of early (E) and late (A) transmitral filling of LV and their ratio (E/A), isovolumetric relaxation time (IVRT) of LV. The RV diastolic function was evaluated in a similar way: the rate of early (Et) and late (At) transtricuspid RV filling and their ratio (Et/At).

Tissue Doppler imaging was used to measure peak early and late diastolic velocities and peak systolic velocity at the lateral mitral annulus (e ', a', s') and the lateral tricuspid annulus (et', a't, s't). The e'/ a', E/e', et'/a't, Et/ et' values and Tei index of the left and right ventricles were calculated. Level of s't $<10 \mathrm{sm} / \mathrm{sec}$ was considered a sign of present RV systolic dysfunction. RV diastolic dysfunction was considered with the ratio Et/At $<0.8$ or $>2.1 \mathrm{and} /$ or the ratio Et/et' $>6$ [14].

\section{Statistical analysis}

Standard STATISTICA 8.0 and SPSS 17.0 software was used for statistical analyses. A case-control study design was used to retrospectively analyze the data of groups with 1 and 2. Qualitative values were presented in absolute numbers (n) and percentage (\%), comparisons between the groups were performed using $\chi 2$ tests. The normality of the distribution was verified using the Kolmogorov-Smirnov test. For a distribution other than normal, all quantitative variables were presented as the median, low and upper quartiles (ME [LQ, UQ]). When comparing quantitative data in two groups, the MannWhitney test was used. Using logistic regression analy- 
sis, we studied the association of possible factors with the presence of RV diastolic dysfunction. Multivariate regression analysis included variables for which the statistical significance in the univariate analysis was less than 0.1 . After a preliminary assessment of the correlation between the identified predictors, several regression models were formed with the inclusion of independent factors. Performance of LV indices for detecting the right ventricle diastolic dysfunction was evaluated through receiver operating characteristic curve analysis. Differences were considered statistically significant at $\mathrm{p}<0.05$.

\section{Results}

\section{Patients'baseline characteristics}

Between February 2017 to November 2018, 765 patients were admitted for coronary artery bypass surgery (CABG). Patients with a recent acute coronary syndrome $(\mathrm{n}=85)$, concomitant valve disease $(\mathrm{n}=53)$, low left ventricle ejection fraction $(<40 \%, \mathrm{n}=124)$ and persistent atrial fibrillation $(\mathrm{n}=82)$ were excluded. Also, we excluded patients with lung diseases accompanied by respiratory failure $(\mathrm{n}=48)$, malignant tumors $(\mathrm{n}=23)$ and poor visualization with ultrasound $(\mathrm{n}=150)$. The inclusion of patients in the study was terminated after the inclusion of a pre-planned number of 200 patients, $7.6 \%$ of patients having RV systolic dysfunction and $46 \%$ of RVDD $(\mathrm{p}<0.001)$.

The patients were divided into 2 groups: group I included patients with RVDD $(\mathrm{n}=92)$ and group II - patients without RVDD ( $\mathrm{n}=108)$. The demographic and clinical characteristics of the 2 groups are detailed in Table I.

Previous myocardial infarction was more prevalent in the group with RVDD ( $\mathrm{p}=0.007)$, more frequently anterior myocardial infarction $(\mathrm{p}=0.001)$. Congestive

Table I. Demographic and clinical characteristic of the study population

\begin{tabular}{|c|c|c|c|}
\hline Parameters & Group 1: RVDD «+» $(\mathrm{n}=92)$ & Group 2: RVDD «-» $(n=108)$ & $\mathbf{P}$ \\
\hline Male, n (\%) & $73(79.35)$ & $80(74.04)$ & 0.380 \\
\hline Age, years & $64[61 ; 69]$ & $63[60 ; 67]$ & 0.138 \\
\hline BMI, $\mathrm{kg} / \mathrm{m}^{2}$ & $28.73[26.12 ; 31.53]$ & $28.25[25.64 ; 30.15]$ & 0.190 \\
\hline Smoking, $\mathrm{n}(\%)$ & $17(21.52)$ & $22(25.88)$ & 0.511 \\
\hline Smoking duration, years & $40[20 ; 45]$ & $40[30 ; 40]$ & 0.854 \\
\hline Arterial hypertension, $\mathrm{n}(\%)$ & $87(94.57)$ & $102(94.44)$ & 0.970 \\
\hline Angina pectoris, $\mathrm{n}(\%)$ & $80(86.96)$ & $94(87.04)$ & 0.986 \\
\hline MI history, $\mathrm{n}(\%)$ & $67(72.83)$ & $59(54.63)$ & 0.007 \\
\hline Anterior myocardial infarction, $\mathrm{n}(\%)$ & $40(43.48)$ & $24(22.22)$ & 0.001 \\
\hline Inferior myocardial infarction, $\mathrm{n}(\%)$ & $27(29.35)$ & $34(31.48)$ & 0.743 \\
\hline RV myocardial infarction, $\mathrm{n}(\%)$ & 0 & $1(0.93)$ & 0.354 \\
\hline Moderate CHF, n (\%) & $25(27.17)$ & $16(14.81)$ & 0.030 \\
\hline Arrhythmias, n (\%) & $7(7.61)$ & $5(4.63)$ & 0.376 \\
\hline Diabetes mellitus, n (\%) & $27(29.35)$ & $27(25)$ & 0.490 \\
\hline Stroke history, n (\%) & $7(7.61)$ & $11(10.19)$ & 0.525 \\
\hline PCI history, $\mathrm{n}(\%)$ & $17(18.48)$ & $18(16.67)$ & 0.736 \\
\hline Chronic lung disease, $\mathrm{n}(\%)$ & $17(18.48)$ & $16(14.81)$ & 0.486 \\
\hline ICA stenosis $>50 \%, \mathrm{n}(\%)$ & $22(23.91)$ & $16(14.81)$ & 0.102 \\
\hline Peripheral arterial disease, $\mathrm{n}(\%)$ & $5(5.43)$ & $3(2.78)$ & 0.339 \\
\hline Multifocal atherosclerosis, n (\%) & $26(28.26)$ & $17(15.74)$ & 0.031 \\
\hline Hyperlipidemia, n (\%) & $65(70.65)$ & $63(58.33)$ & 0.070 \\
\hline NT-proBNP, pg/mL & $66.7[30.7 ; 151.0]$ & $62,75[30.7 ; 97.3]$ & 0.725 \\
\hline Beta-blocker, n (\%) & $90(97.83)$ & $94(87.04)$ & 0.005 \\
\hline Statins, n (\%) & $87(94.57)$ & $104(96.30)$ & 0.556 \\
\hline ACE-I, n (\%) & $69(75.0)$ & $85(78.70)$ & 0.535 \\
\hline
\end{tabular}

The results are expressed as number(\%) or median [low; upper quartiles]; RVDD, right ventricular diastolic dysfunction; ME, median; LQ, lower quartile; UQ, upper quartile; BMI, body mass index; MI, myocardial infarction; RV, right ventricular; CHF, chronic heart failure; PCI, percutaneous coronary intervention; ICA, internal carotid artery; NT-proBNP,N-terminal pro B-type natriuretic peptide; ACE-I, angiotensin converting enzyme inhibitor 
heart failure was observed more frequently in patients with RVDD $(p=0.030)$. Peripheral arterial disease was more often observed in patients with RVDD $(p=0.030)$. $\beta$-blockers were prescribed more often in the group with $\operatorname{RVDD}(\mathrm{p}=0.005)$.
According to ICA, no significant intergroup difference was found in the prevalence of significant coronary arteries stenosis (fig 1): LAD was the most common location $(63 \%$ and $63.9 \%, \mathrm{p}>0.05)$ and three-vessel disease was prevalent $(46.7 \%$ and $49.1 \%, \mathrm{p}>0.05)$.

Table II. Echocardiographic parameters in the coronary artery disease patients with normal and abnormal right ventricle diastolic function

\begin{tabular}{|c|c|c|c|}
\hline Parameters & Group 1: RVDD+ (n=92) & Group 2: RVDD- $(n=108)$ & $\mathbf{p}$ \\
\hline Aorta, $\mathrm{mm}$ & $3.65[3.4 ; 3.9]$ & $3,5[3.4 ; 3.7]$ & 0.138 \\
\hline $\mathrm{LA}, \mathrm{mm}$ & $4.5[4.2 ; 4.9]$ & $4.35[4.1 ; 4.7]$ & 0.140 \\
\hline LV EDD, mm & $5.95[5.5 ; 6.2]$ & $5.4[5.2 ; 6.0]$ & 0.102 \\
\hline LV ESD, mm & $4.0[3.6 ; 4.3]$ & $3.5[3.2 ; 3.8]$ & 0.010 \\
\hline LV EDV, mm & $176.5[147 ; 194]$ & $141[130 ; 180]$ & 0.166 \\
\hline LV ESV, mm & $63[47 ; 75]$ & $48[36 ; 60]$ & 0.070 \\
\hline LV ejection fraction, $\%$ & $61[49 ; 64]$ & $67[58 ; 70]$ & 0.044 \\
\hline LV mass, $\mathrm{g}$ & $312.1[263.9 ; 375.7]$ & $274.2[234 ; 332.8]$ & 0.074 \\
\hline LV mass index, $\mathrm{g} / \mathrm{m}^{2}$ & $153.7[124.3 ; 187.4]$ & $134.0[89.7 ; 153.7]$ & 0.116 \\
\hline LV IVRT, ms & $92[92 ; 98]$ & $92[90 ; 98]$ & 0.418 \\
\hline $\mathrm{E}, \mathrm{cm} / \mathrm{s}$ & $56[44 ; 65]$ & $59[47 ; 68]$ & 0.024 \\
\hline $\mathrm{A}, \mathrm{cm} / \mathrm{s}$ & $70[59 ; 85]$ & $66[59 ; 78]$ & 0.010 \\
\hline $\mathrm{E} / \mathrm{A}$ ratio, & $0.71[0.63 ; 0.83]$ & $0.80[0.71 ; 1.11]$ & $<0.001$ \\
\hline $\mathrm{e}^{\prime}, \mathrm{cm} / \mathrm{s}$ & $9.4[7.5 ; 11.2]$ & $9.0[8.0 ; 11.0]$ & 0.886 \\
\hline $\mathrm{a}^{\prime}, \mathrm{cm} / \mathrm{s}$ & $10.2[8.6 ; 12.3]$ & $9.4[8.2 ; 11.2]$ & 0.139 \\
\hline e'/a' ratio & $0.86[0.68 ; 1.28]$ & $0.91[0.70 ; 1.29]$ & 0.341 \\
\hline $\mathrm{s}^{\prime}, \mathrm{cm} / \mathrm{s}$ & $9.2[8.4 ; 10.6]$ & $9.0[7.7 ; 10.0]$ & 0.268 \\
\hline $\mathrm{E} / \mathrm{e}^{\prime}$ ratio & $5.47[4.62 ; 7.60]$ & $6.22[5.18 ; 7.50]$ & 0.078 \\
\hline LV Tei index & $0.34[0.28 ; 0.43]$ & $0.32[0.22 ; 0.40]$ & 0.017 \\
\hline RV diameter, mm & $2.1[1.8 ; 2.5]$ & $2.0[1.9 ; 2.0]$ & 0.256 \\
\hline RV free wall thickness, mm & $3.5[3.0 ; 4.0]$ & $3.0[3.0 ; 4.0]$ & 0.568 \\
\hline TAPSE, mm & $24[22 ; 24]$ & $22[21 ; 23]$ & 0.129 \\
\hline RV ejection fraction, $\%$ & $52[50 ; 55]$ & $53[51 ; 54]$ & 0.183 \\
\hline RA diameter, mm & $40[33 ; 49]$ & $39[33 ; 51]$ & 0.355 \\
\hline mPAP, $\mathrm{mmHg}$ & $15[12 ; 19]$ & $14[14 ; 16]$ & 0.523 \\
\hline SPAP, mm Hg & $30[28 ; 33]$ & $24[24 ; 27]$ & 0.082 \\
\hline RV Tei index & $0.29[0.24 ; 0.37]$ & $0.30[0.26 ; 0.36]$ & 0.513 \\
\hline $\mathrm{E}_{\mathrm{T}}, \mathrm{cm} / \mathrm{s}$ & $40.5[35 ; 50.5]$ & $45[40 ; 48]$ & 0.059 \\
\hline $\mathrm{A}_{\mathrm{T}}, \mathrm{cm} / \mathrm{s}$ & $47.5[41.0 ; 54.0]$ & $37[31 ; 45]$ & $<0.001$ \\
\hline $\mathrm{E}_{\mathrm{T}} / \mathrm{A}_{\mathrm{T}}$ ratio & $0.75[0.70 ; 1.25]$ & $1.24[0.91 ; 1.45]$ & $<0.001$ \\
\hline $\mathrm{e}^{\prime}, \mathrm{cm} / \mathrm{s}$ & $8.8[7.9 ; 10.3]$ & $10.3[9.0 ; 11.6]$ & $<0.001$ \\
\hline $\mathrm{a}^{\prime}, \mathrm{cm} / \mathrm{s}$ & $14[12.0 ; 15.5]$ & $14.1[12.1 ; 17.0]$ & 0.297 \\
\hline $\mathrm{e}_{\mathrm{T}}^{\prime} / \mathrm{a}_{\mathrm{T}}$, ratio & $0.63[0.57 ; 0.77]$ & $0.73[0.63 ; 0.81]$ & 0.002 \\
\hline $\mathrm{s}_{\mathrm{T}}, \mathrm{cm} / \mathrm{s}$ & $13.2[11.6 ; 14.6]$ & $13.3[12.1 ; 15.0]$ & 0.307 \\
\hline $\mathrm{E}_{\mathrm{T}} / \mathrm{e}_{\mathrm{T}}$, , ratio & $4.43[3.33 ; 6.44]$ & $4.43[3.68 ; 5.04]$ & 0.252 \\
\hline
\end{tabular}

The results are expressed as median [low; upper quartiles]; RVDD,right ventricular diastolic dysfunction; LA, diameter of the left atrium; LV, left ventricular; EDD, end-diastolic dimension; ESD, end-systolic dimension; EDV,end-diastolic volume; ESV,end-systolic volume; IVRT, isovolumic relaxation time; A, late transmitral diastolic filling; E, early transmitral diastolic filling; e', early diastolic mitral annular tissue velocity; a', late diastolic mitral annular tissue velocity; s', systolic mitral annular tissue velocity; Tei,myocardial performance index; RV,right ventricle; TAPSE, tricuspid annular plane systolic excursion; RA,right atrium; mPAP, mean pulmonary artery pressure; SPAP, systolic pulmonary arterial pressure; Eт, early transtricuspid diastolic filling; Ат, late transtricuspid mitral diastolic filling; e'т, early diastolic tricuspid annular tissue velocity; a'T, late diastolic tricuspid annular tissue velocity; s'T, systolic tricuspid annular tissue velocity. 


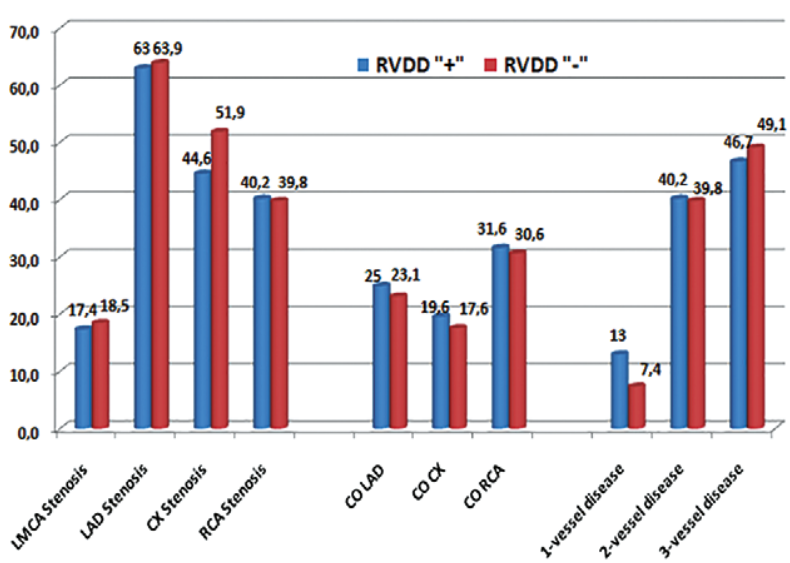

Fig 1. Prevalence and location of coronary atherosclerosis in CAD patients with normal and abnormal right ventricle diastolic function. RVDD, right ventricular diastolic dysfunction; LMCA, left main coronary artery; LAD, left anterior descending; $\mathrm{CX}$, circumflex artery; RCA, right coronary artery; $\mathrm{CO}$, chronic occlusion; CA, coronary artery

\section{Echocardiographic parameters}

The echocardiographic parameters in the CAD patients with normal and abnormal right ventricle diastolic function are detailed in Table II.

In group 1, comparing to group 2, the LV end-systolic dimension was higher $(\mathrm{p}=0.010)$ and LVEF was lower ( $61 \%$ versus $67 \%$, respectively, $\mathrm{p}=0.044$; fig 2 ). In $\mathrm{LV}$, the $\mathrm{E}(\mathrm{p}=0.024)$ and the A were higher in patients with RVDD ( $p=0.010)$. The E/A ratio was significantly higher in the group without RVDD ( $<<0.001$, fig 3$)$. The LV Tei index prevailed in the group with RVDD $(\mathrm{p}=0.017)$.

Among the RV indicators, At was higher and the ratio Et/At was lower in patients with RVDD (both $p<0.001$ ). In the same group of patients, lower e' ${ }_{t}$ velocity $(p<0.001)$ and $e^{\prime} t / a^{\prime} t$ ratio $(p=0.002)$ were noted.

\section{Univariate and multivariate analyses}

We studied association between possible factors with RV diastolic dysfunction using logistic regression analysis (Table III). Multivariate analysis is presented by three models, according to which independent predictors of the RVDD were the increase in age $(\mathrm{p}=0.025)$, "old" MI $(\mathrm{p}=0.018)$, hyperlipidemia $(p=0.029)$, moderate chronic heart failure $(p=0.032)$, peripheral arterial disease $(\mathrm{p}=0.005)$ and decreased LVEF $(\mathrm{p}=0.018)$.

ROC-curves of the studied left ventricular variables (LVEF, ratio E/A and E/e') association with RVDD are presented in fig 4 . The areas under the curves were less than 0.7 (for LVEF - 0.587, for E/A - 0.627, and for E/e' - 0.586), which indicated an unacceptable ability of these indicators to identify RVDD.

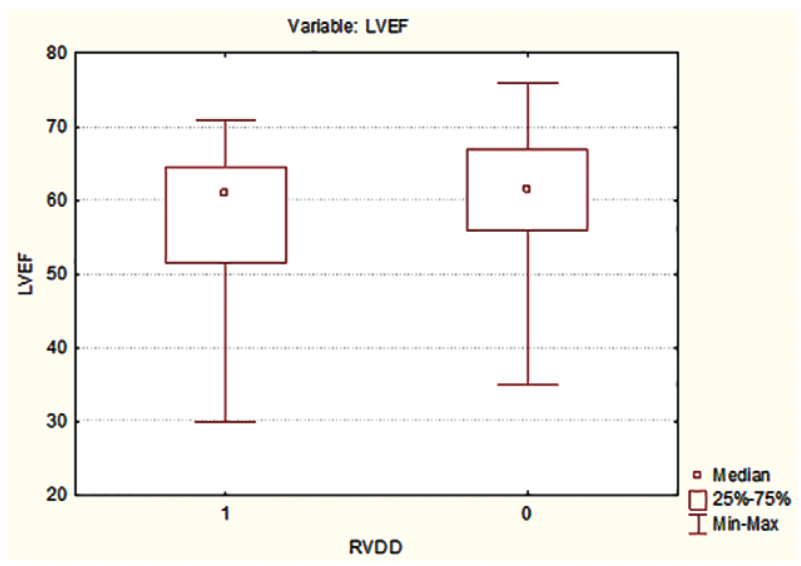

Fig 2. Comparison of left ventricular ejection fraction in groups with and without RVDD. RVDD - right ventricular diastolic dysfunction, 1 - group with RVDD, 0 - group without RVDD, $\mathrm{p}=0.044$ between groups

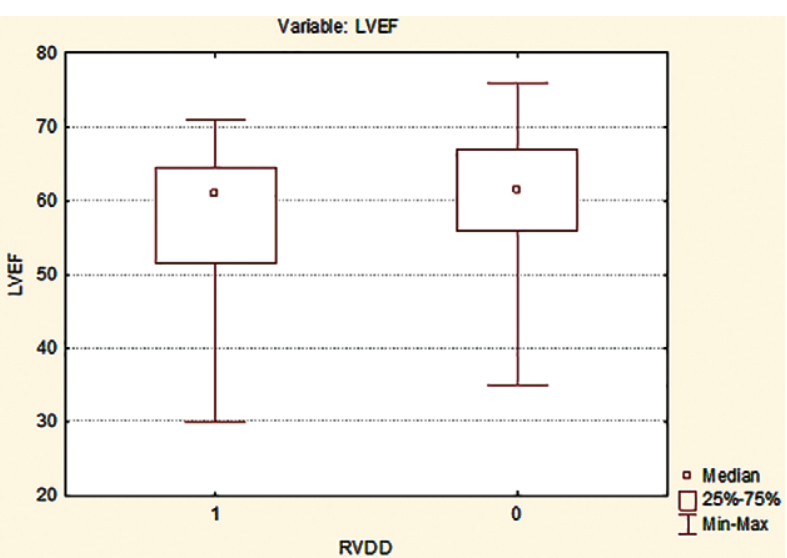

Fig 3. Comparison of ratio E/A in groups with and without RVDD. RVDD - right ventricular diastolic dysfunction, 1 group with RVDD, 0 - group without RVDD, $\mathrm{p}<0.001$ between groups

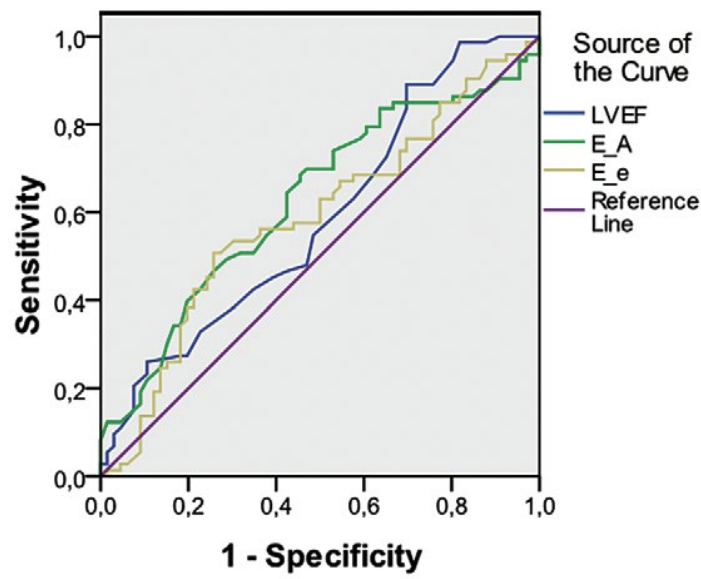

Fig 4. Receiver operating characteristic curve analysis. Performance efficacy of the left ventricle echocardiographic parameters in the RVDD detecting. 
Table III. Univariate and multivariate logistic regression analyses of parameters possibly associated with RV diastolic dysfunction

\begin{tabular}{|c|c|c|}
\hline Parameters & HR $(95 \%$ CI $)$ & $\mathbf{p}$ \\
\hline \multicolumn{3}{|l|}{ Univariate analyses } \\
\hline Age (per year increase) & $0.96(0.92-1.01)$ & 0.147 \\
\hline Myocardial infarction history (yes/no) & $2.22(1.22-4.05)$ & 0.008 \\
\hline Moderate CHF (yes/no) & $2.14(1.06-4.35)$ & 0.033 \\
\hline Multifocal atherosclerosis & $2.11(1.05-4.22)$ & 0.033 \\
\hline Hyperlipidemia(yes/no) & $1.71(0.95-3.11)$ & 0.072 \\
\hline Beta-blocker (yes/no) & $0.15(0.03-0.68)$ & 0.014 \\
\hline Anterior myocardial infarction (yes/no) & $2.69(1.45-4.98)$ & 0.001 \\
\hline LV ESD (per mm increase) & $1.63(1.04-2.57)$ & 0.031 \\
\hline LV ejection fraction (per \% decrease) & $1.04(1.01-1.08)$ & 0.016 \\
\hline RV ejection fraction (per \% decrease) & $1.06(0.98-1.14)$ & 0.103 \\
\hline E (per unit increase) & $1.02(1.0-1.04)$ & 0.033 \\
\hline TAPSE (per mm decrease) & $1.07(0.99-1.15)$ & 0.065 \\
\hline \multicolumn{3}{|l|}{ Multivariate analyses } \\
\hline \multicolumn{3}{|c|}{ Model 1, adjusted for gender,hyperlipidemia, one-vessel disease, $E(p=0.003)$} \\
\hline Age (per year increase) & $1.06(1.01-1.11)$ & 0.025 \\
\hline Myocardial infarction history (yes/no) & $2.15(1.13-4.10)$ & 0.018 \\
\hline \multicolumn{3}{|c|}{ Model 2, adjusted for gender, RV ejection fraction $(\mathrm{p}=0.005)$} \\
\hline Age (per year increase) & $1.06(1.01-1.11)$ & 0.023 \\
\hline Hyperlipidemia(yes/no) & $2.0(1.06-3.78)$ & 0.029 \\
\hline LV EF (per \% decrease) & $1.05(1.01-1.09)$ & 0.018 \\
\hline \multicolumn{3}{|c|}{ Model 3, adjusted for age, gender, smoking, one-vessel disease, TAPSE ( $\mathrm{p}=0.001$ ) } \\
\hline Moderate CHF (yes/no) & $2.22(1.06-4.65)$ & 0.032 \\
\hline Multifocal atherosclerosis (yes/no) & $2.84(1.34-6.0)$ & 0.005 \\
\hline
\end{tabular}

The results are expressed as HR (95\% CI); HR, hazard ratio; CI, confidence interval; RV, right ventricle; $\mathrm{CHF}$, chronic heart failure; $\mathrm{LV}$, left ventricle; ESD, end-systolic diameter; TAPSE, tricuspid annular plane systolic excursion; EF - ejection fraction.

\section{Discussion}

Our investigation showed several findings. First, in stable CAD patients without LV systolic dysfunction, the RVDD occurs in almost half of patients, more frequently comparing to RV systolic dysfunction. Second, RVDD was not associated with the localization and prevalence of coronary artery stenosis. Third, RVDD was associated with an increase in age, a history of myocardial infarction, LV systolic dysfunction, moderate signs of left ventricular heart failure, as well as peripheral arteries disease and dyslipidemia.

Some studies have noted a parallel decrease in both systolic and diastolic RV function [14,15]. At the same time, it was shown that RV diastolic dysfunction can be detected with preserved systolic function in patients with diabetes [16], in patients with congestive heart failure and pulmonary embolism [17].

Since RVDD occurs before RV systolic dysfunction, it is not surprising that RVDD is more frequent in comparison with RV systolic dysfunction, which was noted in our study. Also, among the reasons for the detection of RVDD, a direct adverse effect of pathophysiological factors on the RV myocardium is considered [18]. Since the RV has, in comparison with the LV, a lower mass (ie, lower oxygen consumption), higher compliance and lower pressure, it is possible that similar pathophysiological pathways may have different effects on different parts of the heart [19] and the RV dysfunction may appear earlier than in other structures. In our study age, LV systolic dysfunction and multifocal atherosclerosis were associated with RV dysfunction but the sequence of pathological changes development in RV may be similar. While the influence of LV dysfunction and age on RV function is well known $[9,20]$ the association of RVDD with dyslipidemia and multifocal atherosclerosis is still unclear and requires further research for clarification. Also, the possible lusitropic effect of beta-blockers, which manifested itself in association with RVDD in univariate analysis in our study. However, multivariate analysis did not reveal an independent effect of beta-blockers on RVDD, which is consistent with the results of an experimental study 
on the effect of beta-blockers on LV diastolic function. [21].

In our study, none of the patients had severe RVDD; patients had predominantly RVDD in the form of a relaxation disorder. This is not surprising given the minimal changes in the left and right ventricles in patients. Thus, the criteria we used (the ratio $E_{t} / A_{t}$ and $E_{t} / e_{t}^{\prime}$ ) were sufficient to identify RVDD, their possible limitations (with an increase in pressure in the RV) were not realized in this cohort. Therefore, the use of additional RVDD criteria was not required. As our recent study has shown, this set of RVDD indicators may have prognostic value in certain clinical situations (for example, association with immediate $\mathrm{CABG}$ results [22]).

In patients with $\mathrm{CAD}$, the causes of the RV systolic dysfunction development have been previously studied and were associated with the presence of RV or inferior myocardial infarction [14,15], a three-vessel disease [8] or a single coronary artery lesion [15]. We cannot confirm these associations. Also, in our recent study among the factors independently associated with RV systolic dysfunction were the decrease of LV ejection fraction, but not individual coronary arteries lesions, the number of affected coronary arteries or the presence and localization of myocardial infarction [24], similar with other studies evaluating RV systolic dysfunction [5,6]. Possible causes of such discrepancies may be the dependence of the RV systolic dysfunction presence on the collateral vessels development (their insufficient development was associated with systolic RV dysfunction [15]) and the level of coronary artery lesions (systolic RV dysfunction was more often observed in proximal lesions [6]). Systolic RV dysfunction may be associated with a transient perfusion defect in the LV inferior-lateral segments, rather than a stable perfusion defect [23].

Tajima et al [8] revealed a relationship between the number of affected coronary arteries and the presence of RV systolic dysfunction in patients after myocardial revascularization. In the group with RV systolic dysfunction CABG was performed more often [8]. Since after open heart surgery, there is a decrease in the RV systolic function [25] and this can explain the results obtained by the authors. That pattern could not be confirmed in our study, and in some others [5,6].

We found an association of RV diastolic dysfunction with deterioration of LV pumping function, as well as with previous anterior MI, but not with damage to the coronary arteries. Therefore, it can be assumed that the development of LV ischemic cardiomyopathy and secondary changes in RV are the leading mechanisms of RVDD development (possibly due to interventricular interactions) but not direct RV ischemic changes.
The present study has limitations and the results should be interpreted cautiously. First, only the Caucasian population was included, therefore, its results cannot be extended to other ethnic groups. Second, patients with concomitant diseases were excluded from the study, which reduces the generalization of the available results, especially in patients with lung diseases and valvular pathology. Third, this study is cross-sectional in nature; therefore, reverse causation is possible; future studies should prospectively assess these associations. Another limitation is the relatively small number of included patients. Although we were able to obtain statistically significant results, they need to be confirmed in studies with a large number of patients. Finally, the definition of the $\mathrm{RV}$ function was based on the standard indexes of RV systolic and diastolic function without second level techniques (speckle-tracking RV measurement, 3D echocardiography), which have been used in recent years [26]. However, new technologies, such as global longitudinal strain $(3 \%)$ and 3D echocardiography $(1 \%)$, have rarely been used to quantify RV function in clinical practice [27]. Therefore, the use of traditional RV indicators, in our opinion, can be justified currently. However, in the future it is rational to conduct studies using new technologies for assessing RV systolic and diastolic functions.

As we showed that in patients with stable CAD with preserved or slightly reduced LV ejection fraction, RVDD develops earlier than systolic dysfunction, and this may lead to better risk stratification of patients, since an unfavorable predictive value of RVDD in CABG has been shown both in patients with $\mathrm{EF} \leq 35 \%$ [28] and in patients with preserved LVEF [22]. The prognostic value of RV diastolic dysfunction in other categories of patients with coronary artery disease remains unclear.

In conclusion, in patients with stable $\mathrm{CAD}$, the right ventricle diastolic dysfunction is much more common than its systolic dysfunction. The presence of RV diastolic dysfunction was associated with an increase in age, a history of myocardial infarction, LVEF decrease, moderate signs of left ventricular heart failure, as well as peripheral arteries disease and dyslipidemia. No association of RV diastolic dysfunction with the localization and prevalence of coronary artery stenosis was revealed. The clinical and prognostic value of RV diastolic dysfunction in patients with chronic coronary syndrome remains to be studied.

\section{Conflict of interest: none}

\section{References}

1. Akazawa Y, Fujioka T, Kühn A, et al. Right Ventricular Diastolic Function and Right Atrial Function and Their Re- 
lation With Exercise Capacity in Ebstein Anomaly. Can J Cardiol 2019;35:1824-1833.

2. Polito MV, Stoebe S, Galasso G, et al. Analysis of Regional Right Ventricular Function by Tissue Doppler Imaging in Patients with Aortic Stenosis. J Cardiovasc Echogr 2019;29:111-118.

3. Tello K, Seeger W, Naeije R, et al. Right heart failure in pulmonary hypertension: Diagnosis and new perspectives on vascular and direct right ventricular treatment. $\mathrm{Br} \mathrm{J}$ Pharmacol 2021;178:90-107.

4. Cameli M, Pastore MC, Henein MY, Mondillo S. The left atrium and the right ventricle: two supporting chambers to the failing left ventricle. Heart Fail Rev 2019;24:661-669.

5. Keskin M, Uzun AO, Hayıroğlu Mİ, Kaya A, Çınar T, Kozan Ö. The association of right ventricular dysfunction with in-hospital and 1-year outcomes in anterior myocardial infarction. Int J Cardiovasc Imaging 2019;35:77-85.

6. Pouleur AC, Rousseau MF, Ahn SA, et al. Right Ventricular Systolic Dysfunction Assessed by Cardiac Magnetic Resonance Is a Strong Predictor of Cardiovascular Death After Coronary Bypass Grafting. Ann Thorac Surg 2016;101:2176-2184.

7. Surkova E, Muraru D, Genovese D, Aruta P, Palermo C, Badano LP. Relative Prognostic Importance of Left and Right Ventricular Ejection Fraction in Patients With Cardiac Diseases. J Am Soc Echocardiogr 2019;32:1407-1415.e3.

8. Tajima M, Nakayama A, Uewaki R, Mahara K, Isobe M, Nagayama M. Right ventricular dysfunction is associated with exercise intolerance and poor prognosis in ischemic heart disease. Heart Vessels 2019;34:385-392.

9. Gavazzoni M, Badano LP, Vizzardi E, et al. Prognostic value of right ventricular free wall longitudinal strain in a large cohort of outpatients with left-side heart disease. Eur Heart J Cardiovasc Imaging 2020;21:1013-1021.

10. Murch SD, La Gerche A, Roberts TJ, Prior DL, MacIsaac AI, Burns AT. Abnormal right ventricular relaxation in pulmonary hypertension. Pulm Circ 2015;5:370-375.

11. Miao Y, Zhang W, Zhongi Y, Ma X. Diastolic function of the right ventricle is impaired in experimental type 2 diabetic rat models. Turk J Med Sci 2014;44:448-453.

12. Lang RM, Badano LP, Mor-Avi V, et al. Recommendations for cardiac chamber quantification by echocardiography in adults: an update from the American Society of Echocardiography and the European Association of Cardiovascular Imaging. Eur Heart J Cardiovasc Imaging 2015;16:233270.

13. Rudski LG, Lai WW, Afilalo J, et al. Guidelines for the echocardiographic assessment of the right heart in adults: a report from the American Society of Echocardiography endorsed by the European Association of Echocardiography, a registered branch of the European Society of Cardiology, and the Canadian Society of Echocardiography. J Am Soc Echocardiogr 2010;23:685-713.

14. Mukhaini M, Prashanth P, Abdulrehman S, Zadjali M. Assessment of right ventricular diastolic function by tissue Doppler imaging in patients with acute right ventricular myocardial infarction. Echocardiography 2010;27:539-543.
15. Missiri AM., Guindy RR. Echocardiographic assessment of right ventricular functions in patients with proximal right coronary artery chronic total occlusion. Int J Cardiovasc Imaging 2016;32:895-903.

16. Karamitsos, TD, Karvounis HI, Dalamanga EG, et al. Early diastolic impairment of diabetic heart: the significance of right ventricle. Int J Cardiol 2007;114:218-223.

17. Gromadziński L, Targoński R, Pruszczyk P. Assessment of right and left ventricular diastolic functions with tissue Doppler echocardiography in congestive heart failure patients with coexisting acute pulmonary embolism. Adv Clin Exp Med 2014;23:371-376.

18. Linssen PBC, Veugen MGJ, Henry RMA, et al. Associations of (pre)diabetes with right ventricular and atrial structure and function: the Maastricht Study. Cardiovasc Diabetol 2020;19:88.

19. Sanz J, Sanchez-Quintana D, Bossone E, Bogaard HJ, Naeije R. Anatomy, function, and dysfunction of the right ventricle: JACC state-of-the-art review. J Am Coll Cardiol 2019;73:1463-1482.

20. D'Andrea A, Vriz O, Carbone A, et al. The impact of age and gender on right ventricular diastolic function among healthy adults. J Cardiol 2017;70:387-395.

21. Bamaiyi AJ, Norton GR, Peterson V, Norman G, Mojiminiyi FB, Woodiwiss AJ. Limited Impact of $\beta$-Adrenergic Receptor Activation on Left Ventricular Diastolic Function in Rat Models of Hypertensive Heart Disease. J Cardiovasc Pharmacol 2018;72:242-251.

22. Sumin AN, Korok EV, Sergeeva TJ. Preexisting Right Ventricular Diastolic Dysfunction and Postoperative Cardiac Complications in Patients Undergoing Nonemergency Coronary Artery Bypass Surgery. J Cardiothorac Vasc Anesth 2020. doi:10.1053/j.jvca.2020.09.100.

23. Kim J, Di Franco A, Seoane T, et al. Right Ventricular Dysfunction Impairs Effort Tolerance Independent of Left Ventricular Function Among Patients Undergoing Exercise Stress Myocardial Perfusion Imaging. Circ Cardiovasc Imaging 2016;9:e005115.

24. Sumin AN, Korok EV, Sergeeva TYu. Moderate right ventricular systolic dysfunction in patients with chronic coronary syndrome - what determines it? Complex Issues of Cardiovascular Diseases 2019;8(4S):51-61.

25. Chinikar M, Rafiee M, Aghajankhah M, et al. Right ventricular dysfunction and associated factors in patients after coronary artery bypass grafting. ARYA Atheroscler 2019;15:99-105.

26. Mandoli GE, Cameli M, Novo G, et al; Right ventricular function after cardiac surgery: the diagnostic and prognostic role of echocardiography. Heart Fail Rev 2019;24:625635.

27. Schneider M, Aschauer S, Mascherbauer J, et al. Echocardiographic assessment of right ventricular function: current clinical practice. Int J Cardiovasc Imaging 2019;35:49-56.

28. Jin Y, Wang H, Wang Z, Jiang H, Tao D, Wu H. The evaluation of preoperative right ventricular diastolic dysfunction on coronary artery disease patients with left ventricular dysfunction. Echocardiography 2014;31:1259-1264. 